\title{
Transverse Oscillation Vector Velocity Estimation using a Phased Array Transducer
}

Marcher, Jønne; Pihl, Michael Johannes; Seerup, Gert; Haugaard, Per; Nikolov, Svetoslav Ivanov; Jensen, Jørgen Arendt

\section{Published in:}

Proceedings of IEEE International Ultrasonics Symposium

Link to article, DOI:

10.1109/ULTSYM.2012.0474

Publication date:

2012

Link back to DTU Orbit

Citation (APA):

Marcher, J., Pihl, M. J., Seerup, G., Haugaard, P., Nikolov, S. I., \& Jensen, J. A. (2012). Transverse Oscillation Vector Velocity Estimation using a Phased Array Transducer. In Proceedings of IEEE International Ultrasonics Symposium (pp. 1890-1893). IEEE. https://doi.org/10.1109/ULTSYM.2012.0474

\section{General rights}

Copyright and moral rights for the publications made accessible in the public portal are retained by the authors and/or other copyright owners and it is a condition of accessing publications that users recognise and abide by the legal requirements associated with these rights.

- Users may download and print one copy of any publication from the public portal for the purpose of private study or research.

- You may not further distribute the material or use it for any profit-making activity or commercial gain

- You may freely distribute the URL identifying the publication in the public portal 


\title{
Transverse Oscillation Vector Velocity Estimation using a Phased Array Transducer
}

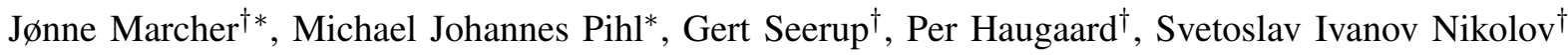 \\ and Jørgen Arendt Jensen* \\ ${ }^{*}$ Center for Fast Ultrasound Imaging, Department of Electrical Engineering, \\ Technical University of Denmark, DK-2800 Kgs. Lyngby, Denmark \\ ${ }^{\dagger}$ BK Medical ApS, Mileparken 34, DK-2730 Herlev, Denmark
}

\begin{abstract}
The Transverse Oscillation method has shown its commercial feasibility, providing the user with 2D velocity information. Todays implementation on commercial ultrasound platforms only support linear array transducers and are limited in depth. Extending the implementation to a phased array transducer, vector velocity echocardiography will become possible. This paper describes the general modification made on the BK Medical 2202 Pro Focus UltraView using a 64 element phased array transducer and the simulations and measurements performed. The results show that velocities can be obtained at depths even greater than $100 \mathrm{~mm}$. Tests at depths of $72 \mathrm{~mm}$ and $82 \mathrm{~mm}$ with a peak velocity of $0.5 \mathrm{~m} / \mathrm{s}$, showed a relative mean bias $\tilde{B}_{v_{x}}$ that varied from $0 \%$ and to $21 \%$ and a relative mean standard deviation $\tilde{\sigma}_{v_{x}}$ that varied from $18 \%$ and to 51 $\%$. The investigation showed an increasing bias with respect to depth, which leaves room for optimization. Despite the bias, the method has shown to work and produce reliable results, and 2D velocity estimates are provided within the entire color-box down to a depth of more than $100 \mathrm{~mm}$ making vector velocity imaging possible in the entire heart.
\end{abstract}

\section{INTRODUCTION}

For many years medical ultrasound velocity imaging has been used to diagnose cardiovascular diseases. Many techniques have been proposed and used through out the years. This paper is focusing on the Transverse Oscillation method (TO) suggested by [1], [2]. This method uses a transverse oscillating field to estimate both the axial and lateral velocity component, which has similarities to methods proposed by both [3] and [4]. Previous investigations have used the TO method in combination with a phased array transducer to do elastography [5]. The focus of this paper will be on the commercial implementation of blood velocity estimation.

The TO method has been used for in-vivo imaging [6], providing the user with $2 \mathrm{D}$ velocity information (Lateral and axial velocity). However, the current implementation on commercial ultrasound platforms is limited to linear array transducers. By extending the implementation to a phased array transducer [7], [8], vector velocity echocardiography will become possible. Field II simulations [9], [10] and flow rig measurements are used to investigate the feasibility and the possibility of implementing the TO method using a phased array transducer for commercial scanner purposes. Measurements are performed using a preliminary implementation on a BK Medical ultrasound scanner platform. Through this the performance of the implementation will be tested for different

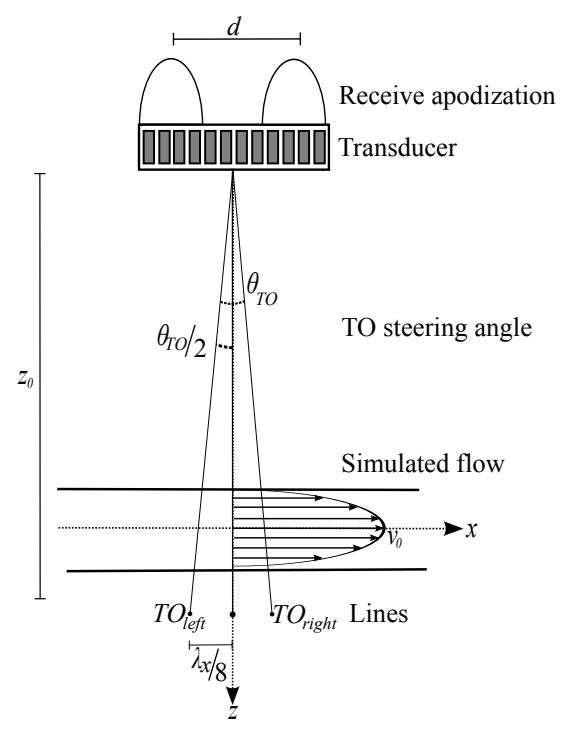

Fig. 1. Description of the relation between beam steering through time delays profiles and apodization profiles to obtain sampling at $\lambda_{x} / 4$ wave length. Courtesy of PhD Michael Johannes Pihl

depths, steering angles $(\theta)$, and flow to transducer angles $\left(\theta_{f 2 t}\right)$.

\section{Measurement Setup}

Previous investigations have shown, through simulations in Field II and implementation on the experimental scanner SARUS [11], that an implementation using a phased array transducer is possible [8]. These investigations were, however, performed with a 128 element $\lambda / 2$-pitch transducer. Vector velocity echocardiography requires a transducer with a small footprint for scanning between the ribs. A 64 element phased array transducer with a pitch of $0.33 \mathrm{~mm}$ is chosen for the investigation. The $3 \mathrm{MHz}, 75 \%$ bandwidth, 64 element transducer is simulated using Field II and the results are used to verify the implementation on the BK Medical ultrasound scanner. Three beams are needed to produce estimates of the velocity, see Fig. 1. A normal focused emission is made and the three beams are focused in parallel during receive processing. The first beam is parallel to the steering angle (the center beam) and is used for making the axial velocity estimate $v_{z}$ using conventional color flow mapping (CFM). The second 
and third beam (the left beam and the right beam) are placed symmetrically around the center beam and are placed at an inter-spatial distance of a quarter of the lateral wavelength, $\lambda_{x} / 4$. The quarter of a wave length ensures that the two beams are each others in-phase/quadrature pair, making it possible to estimate the transverse velocity $v_{x}$ 's direction. The angle between the beams are determined using [7], [8]:

$$
\theta_{T O}=2 \arctan \frac{\lambda_{x} / 8}{z_{0}}=2 \arctan \frac{\lambda_{z}}{4 d},
$$

where $d$ is the distance between the apodization peaks and $z_{0}$ the depth according to Fig. 1. $\lambda_{z}$ is the axial wave length given by $\lambda_{z}=\frac{c}{f_{0}}$, where $c$ is the speed of sound and $f_{0}$ the center frequency of the pulse.

To obtain the double oscillating field required by the method, an apodization having two distinct peaks is employed. The distance $d$ between the apodization peaks, see Fig. 1, is directly related to the lateral oscillation period when using the Fraunhofer far-field approximation:

$$
\lambda_{x}=2 \lambda_{z} \frac{z}{d},
$$

where $z$ is the depth. So by varying the distance between the peaks $\lambda_{x}$ can be controlled. Increasing the width of the peaks will allow for more signal energy to be received. However, this will also narrow the field, leading to a decrease in the performance of the estimator.

The ultrasound scanner used is a BK Medical 2202 Pro Focus UltraView, equipped with a BK UA2227 research interface that allows a PC to acquire data from the scanner [12]. This is performed through a camera link interface and an Ethernet connection for data transport. The data are stored on the PC and processed off-line using Matlab.

To make vector flow imaging (VFI) using the ultrasound scanner, the usecase of the scanner is changed. The usecase is the information file containing all information about the scan. Modifying the usecase will make it possible to change the scanner setup to fit the scan purpose. A conventional (CFM) usecase is modified. The position and size of the color-box results in a field-of-interest equal to the one displayed in Fig. 2. Within this color-box there are 22 steering angles varying from -12.2 degrees to 12.2 degrees. For each steering angle there are beamformed lines for the TO estimator, which are separated by $\lambda_{x} / 4$ [7]. These lines are controlled by custom time delay profiles, which are written to the scanner. To obtain the double oscillating field a receive apodization using a Tukey window of 16 elements, with the peaks separated by 48 elements is used to make the two distinct apodization peaks.

The implementation is tested at different depths, steering angles $\theta$, and flow angles $\theta_{f 2 t}$ in a calibrated flow rig. The flow rig consists of a water tank containing a rubber tube with a radius of $6 \mathrm{~mm}$, which is penetrable by ultrasound. The tube has a straight inlet, sufficiently long to obtain laminar flow with parabolic shape. The volume flow is measured using a Danfoss MAGFLO Mag 3000 flow meter and through this, the true velocity profile in the vessel is estimated. The investigation is performed at two depths; $72 \mathrm{~mm}$ and $82 \mathrm{~mm}$

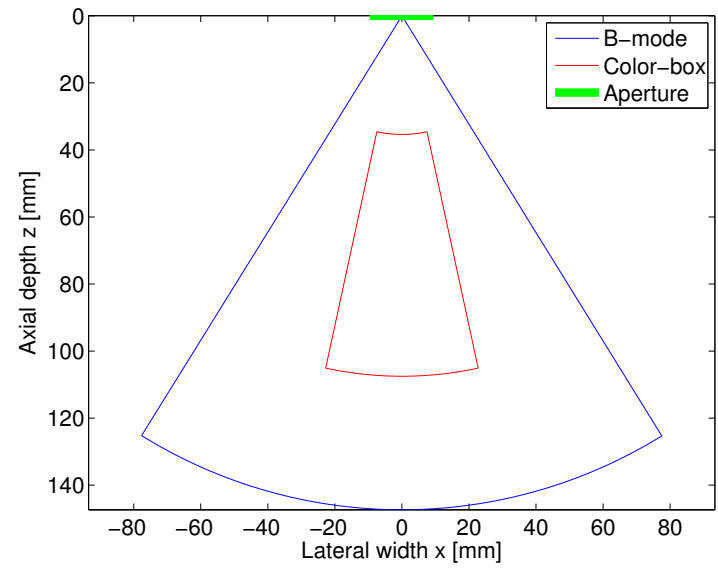

Fig. 2. Resulting Scan Area. The B-mode data is acquired within the the blue line and the velocity data is acquired within the red line. Notice that with a phased array transducer it is possible to obtain a field-of-view greater than the aperture size.

TABLE I

INVESTIGATION PARAMETERS

\begin{tabular}{ll}
\hline Transducer parameters & Value \\
\hline Transducer & Phased array \\
Number of elements & 64 \\
Center frequency & $3 \mathrm{MHz}$ \\
Bandwidth & $75 \%$ \\
Pitch & $0.3 \mathrm{~mm}$ \\
Kerf & approx. $0.04 \mathrm{~mm}$ \\
\hline Setup parameters & $120 \mathrm{MHz}$ \\
\hline Simulation sampling frequency & $12 \mathrm{MHz}$ \\
Scanner sampling frequency & $1.4 \mathrm{kHz}$ and $3.6 \mathrm{kHz}$ \\
Pulse repetition frequency & $1480 \mathrm{~m} / \mathrm{s}$ \\
Speed of sound & $0.5 \mathrm{~m} / \mathrm{s}$ \\
Maximum blood velocity & 6 \\
No. of transmit cycles & $160 \mathrm{~mm}$ \\
Transmit focus (radial depth) & 20 \\
Transmit F\# & {$[7282]$ mm } \\
Center of vessel & $-12: 12 \mathrm{degrees}$ \\
Steering angle & {$[020] \mathrm{degrees}$} \\
Flow-to-transducer angle & Square \\
Transmit apodization shape & Tukey \\
Receive apodization shape & 48 elements \\
Space between peaks & 16 elements \\
Apodization width & 16 \\
Number of emissions per estimate & \\
\hline
\end{tabular}

with two different flow to transducer angles $\theta_{f 2 t}$ of 0 degrees and 20 degrees. The parameters are listed in Table I and are the same for simulations and measurements.

\section{RESULTS}

The main focus is on the transverse velocity $v_{x}$, since the conventional autocorrelation estimator for the axial velocity $v_{z}$ already is well described. There is made no effort to reject velocity estimates outside the vessel boundaries. A mean-subtraction filter is used for making the stationary echocanceling. For each case there are recorded 100 frames, equal to 100 estimates of the velocity. For all investigations a peak velocity $v_{0}$ of $0.5 \mathrm{~m} / \mathrm{s}$ is used. For $\theta_{f 2 t}=0$ degrees the pulse repetition frequency $f_{p r f}$ is $1.4 \mathrm{kHz}$, which is increased to $3.6 \mathrm{kHz}$ at $\theta_{f 2 t}=20$ degrees due the larger axial velocity 


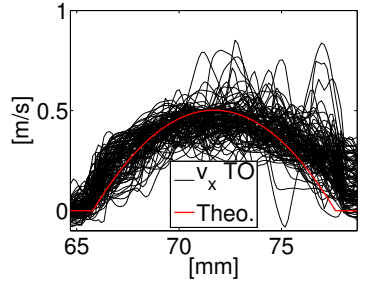

(a) Estimated transverse velocity profiles

Fig. 3. Result of velocity estimation at 0 degrees $\theta_{f 2 t}$

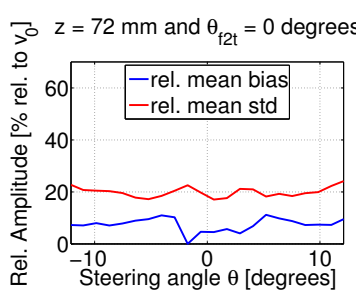

(a)

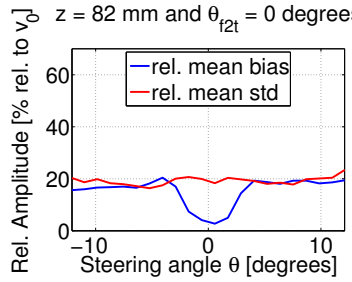

(c)

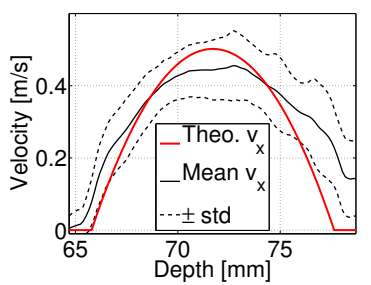

(b) Mean \pm std profile

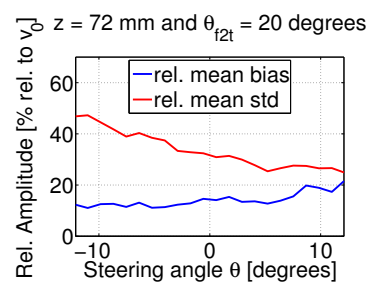

(b)

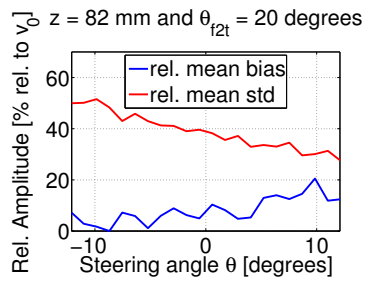

(d)
Fig. 4. Results at $72 \mathrm{~mm}$ and $82 \mathrm{~mm}$

component. The obtained RF data form the research interface are post-processed using Matlab.

Velocity profiles estimated using the TO estimator are shown in Fig. 3a. The mean profile \pm one standard deviation is shown in Fig. $3 \mathrm{~b}$. The relative mean bias $\tilde{B}_{v_{x}}$ is found by estimating the bias by subtracting the mean from the true velocity profile and then average the bias over the profile. The mean bias is made relative to the true peak velocity $v_{0}$ :

$$
\tilde{B}_{v_{x}}=\frac{1}{v_{0}} \frac{1}{\left(n_{z_{1}}-n_{z_{2}}\right)} \sum_{n_{z}=n_{z_{1}}}^{n_{z_{2}}} v_{\text {true }}\left(n_{z}\right)-\bar{v}\left(n_{z}\right),
$$

where $n_{z}$ are the discrete samples in depth, $v_{\text {true }}\left(n_{z}\right)$ is the true velocity profile and $\bar{v}\left(n_{z}\right)$ is the estimated mean velocity profile.

The same procedure is made for the standard deviation providing the relative mean standard deviation, $\tilde{\sigma}_{v_{x}}$. The evaluation is performed for all steering angles, and the results are shown in Fig. 4a.

The performance measures $\tilde{B}_{v_{x}}$ and $\tilde{\sigma}_{v_{x}}$ are found at the two depths of interest, $72 \mathrm{~mm}$ and $82 \mathrm{~mm}$. The results are shown in Fig. 4a, 4b, and 4c, 4d respectively and listed in Table II.

Combining the transverse velocity $v_{x}$ and the axial velocity $v_{z}$ yields an estimate of the true velocity $|\vec{v}|$ and an estimate of the flow to transducer angle $\theta_{f 2 t}$. The frames are time averaged over four frames equal to a persistence of four. The
TABLE II

INVESTIGATION RESULTS

\begin{tabular}{lcccc} 
Depth & $\theta_{f 2 t}$ & Figure & $\tilde{B}_{v_{x}}$ & $\tilde{\sigma}_{v_{x}}$ \\
\hline $72 \mathrm{~mm}$ & 0 degrees & $4 \mathrm{a}$ & $0-12 \%$ & $18-22 \%$ \\
& 20 degrees & $4 \mathrm{~b}$ & $11-20 \%$ & $25-47 \%$ \\
\hline $82 \mathrm{~mm}$ & 0 degrees & $4 \mathrm{c}$ & $3-21 \%$ & $16-19 \%$ \\
& 20 degrees & $4 \mathrm{~d}$ & $0-21 \%$ & $27-51 \%$
\end{tabular}

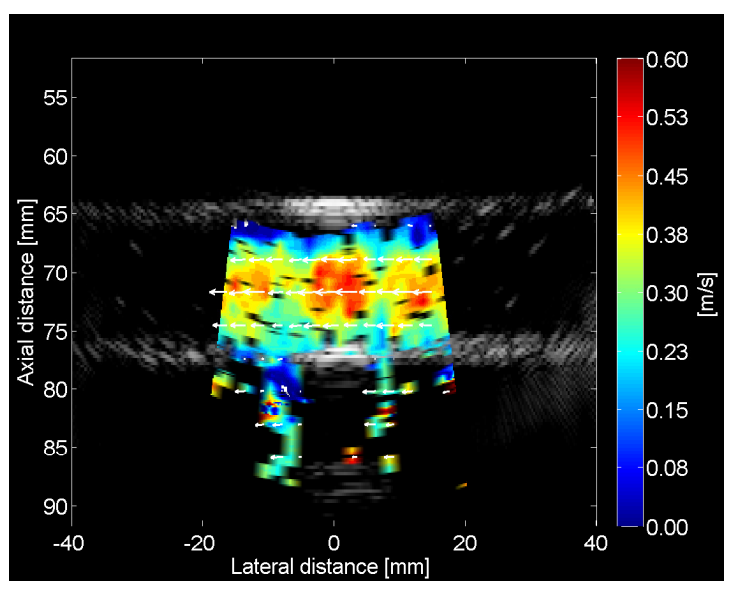

(a) $72 \mathrm{~mm}$

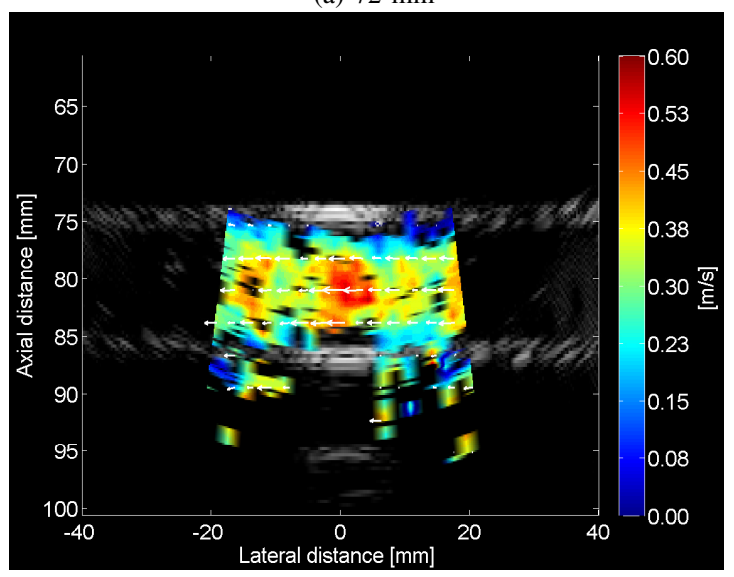

(b) $82 \mathrm{~mm}$

Fig. 5. Flow images at a depth of $72 \mathrm{~mm}$ and $82 \mathrm{~mm}$ with $\theta_{f 2 t}=0$ degrees

data have been scan-converted to rectangular coordinates and interpolated to fit a 512 x 512 pixel window. A simple power ratio between the power before $P_{r f}$ and after echo canceling $P_{e c}$ is used to discriminate between inside and outside of the vessel. This is made by setting velocity estimates to zero for $\frac{P_{e c}}{P_{r f}}$ values below a certain threshold. The obtained VFI image for the implementation is shown in Fig. 5.

\section{Discussion}

It is important to state that this is the first setup with the scanner in VFI mode using this phased array transducer. From Table II and from Fig. 4 it is seen, that for the two individual cases with $\theta_{f 2 t}=0$ degrees $\theta_{f 2 t}=20$ degrees $\tilde{\sigma}_{v_{x}}$ does not differ significantly for the different depths. The error in the estimated velocity is not effected by the increased depth, but is more dependent on the combination of steering angle 


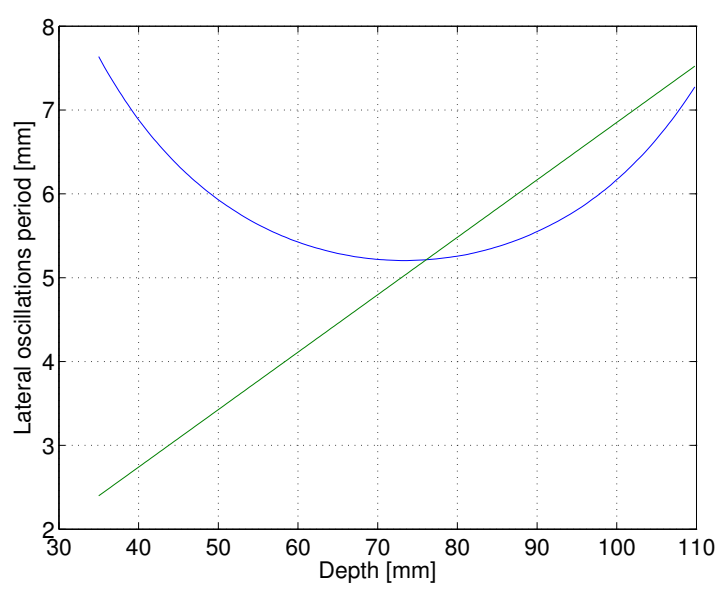

Fig. 6. Fraunhofer approximation and simulated lateral wavelength $\lambda_{x}$

and flow-to-transducer angle. The bias changes as a function of combined steering angle and flow-to-transducer angle and depth. Which shows that the mean of the velocity estimates deviates from the true velocity, which is due to an erroneous scaling of the autocorrelations.

In the flow images in Fig. 5 the largest velocities are found at the center of the tube, with decreasing velocities when moving toward the boundaries of the tube, as expected. Furthermore, it is also seen that the the velocity estimates, hence the bias, change with the steering angle, as seen from Fig. 4. Notice the precise direction of the arrows that points correctly along the flow direction.

The results of the implementation have shown room for optimization, since the bias can be decreased along with, to some extend, the standard deviation.

Simulations and practical experiments have shown that the lateral wavelength, $\lambda_{x}$, does not perfectly apply to the theoretical Fraunhofer far-field approximation. As seen from (2) the Fraunhofer far-field approximation suggest a linear relation between lateral wave length and depth. For this given setup the lateral wavelength has been simulated and compared to the theoretical approximation. The simulation parameters are chosen carefully to match the real case. By minimizing the left-half plane leakage of the Fourier transform of the point spread function, the simulated lateral wave length is found. By minimizing the leakage into the left half plane the bias and standard deviation is decreased when estimating velocities. The depth has been varied from $35 \mathrm{~mm}$ to $110 \mathrm{~mm}$ and at each depth the simulated and theoretical wave length are compared in Fig. 6. The green line represents the theoretical approximation of the lateral wavelength and the blue line represents the found wavelength estimated from the simulated point spread function. Fig. 6 shows that the actual wave length does not follow the linear relation to depth, which results in a difference between the theoretical and actual wavelength.

The beamformation of the TO lines and their relative placement is dependent on the lateral oscillation period. The fields and thereby the velocity estimates can be optimized by simulating the TO fields and then optimize the focusing and scaling of the velocity estimates. This can potentially reduce both bias and standard deviation.

\section{CONCLUSION}

The results show that the implementation of the Transverse Oscillation method on the commercial platform using a 64 element phased array transducer works. It is shown that velocities are found down to depths of $100+\mathrm{mm}$, which substantiate the scope of making velocity estimation in deep regions, like echocardiography, possible. Furthermore, it is seen that with calibration the method will yield acceptable and reliable velocity estimates.

\section{ACKNOWLEDGMENTS}

This work was made in a collaboration between Center for Fast Ultrasound Imaging at the Technical University of Denmark and BK Medical ApS.

\section{REFERENCES}

[1] J. A. Jensen and P. Munk, "A New Method for Estimation of Velocity Vectors," IEEE Trans. Ultrason., Ferroelec., Freq. Contr., vol. 45, pp. 837-851, 1998.

[2] J. A. Jensen, "A New Estimator for Vector Velocity Estimation," IEEE Trans. Ultrason., Ferroelec., Freq. Contr., vol. 48, no. 4, pp. 886-894, 2001.

[3] M. E. Anderson, "Multi-dimensional velocity estimation with ultrasound using spatial quadrature," IEEE Trans. Ultrason., Ferroelec., Freq. Contr., vol. 45, pp. 852-861, 1998.

[4] C. Sumi, "Displacement vector measurement using instantaneous ultrasound signal phase - multidimensional autocorrelation and Doppler methods," IEEE Trans. Ultrason., Ferroelec., Freq. Contr., vol. 55, no. 1, pp. 24-43, 2008.

[5] H. Liebgott, A. Basarab, S. Marincas, O. Bernard, and D. Friboulet, "Tangential oscillations for motion estimation in echocardiography," in Proc. IEEE Ultrason. Symp., nov. 2008, pp. $1761-1764$.

[6] J. Udesen, M. B. Nielsen, K. R. Nielsen, and J. A. Jensen, "Examples of in-vivo blood vector velocity estimation," Ultrasound Med. Biol., vol. 33, pp. 541-548, 2007.

[7] M. J. Pihl and J. A. Jensen, "Transverse oscillations for phased array vector velocity imaging," in Proc. IEEE Ultrason. Symp., 2010, pp. 1323-27.

[8] M. J. Pihl, J. Marcher, and J. A. Jensen, "Phased array vector velocity estimation using tranverse oscillations," IEEE Trans. Ultrason., Ferroelec., Freq. Contr., vol. In press, 2012.

[9] J. A. Jensen, "Field: A Program for Simulating Ultrasound Systems," Med. Biol. Eng. Comp., vol. 10th Nordic-Baltic Conference on Biomedical Imaging, Vol. 4, Supplement 1, Part 1, pp. 351-353, 1996.

[10] J. A. Jensen and N. B. Svendsen, "Calculation of Pressure Fields from Arbitrarily Shaped, Apodized, and Excited Ultrasound Transducers," IEEE Trans. Ultrason., Ferroelec., Freq. Contr., vol. 39, pp. 262-267, 1992.

[11] J. A. Jensen, H. Holten-Lund, R. T. Nielson, B. G. Tomov, M. B. Stuart, S. I. Nikolov, M. Hansen, and U. D. Larsen, "Performance of SARUS: A Synthetic Aperture Real-time Ultrasound System," in Proc. IEEE Ultrason. Symp., Oct. 2010, pp. 305-309.

[12] M. C. Hemmsen, S. I. Nikolov, M. M. Pedersen, M. J. Pihl, M. S. Enevoldsen, J. M. Hansen, and J. A. Jensen, "Implementation of a versatile research data acquisition system using a commercially available medical ultrasound scanner," IEEE Trans. Ultrason., Ferroelec., Freq. Contr., vol. 59, no. 7, pp. 1487-1499, 2011. 